\title{
ORGANIZATION OF THE R CHROMOSOME \\ REGION IN MAIZE
}

Progress Report

for

Sept. 1, 1976 - Oct. 31, 1977

J. L. Kermicle

University of Wisconsin

Madison, Wisconsin 53706

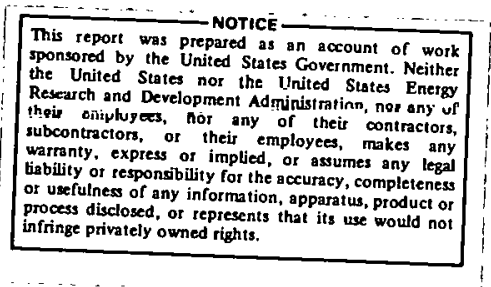

Ju1y 1977

THE U.S. ENERGY RESEARCH AND DEVELOPMENT ADMINISTRATION

Contract EY-76-S-02-1300

$\varepsilon \beta$

DISTRIBUTION OF THIS DOCUMENT IS UNLIMITED

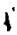

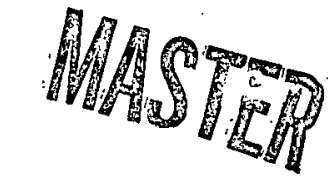




\section{DISCLAIMER}

This report was prepared as an account of work sponsored by an agency of the United States Government. Neither the United States Government nor any agency Thereof, nor any of their employees, makes any warranty, express or implied, or assumes any legal liability or responsibility for the accuracy, completeness, or usefulness of any information, apparatus, product, or process disclosed, or represents that its use would not infringe privately owned rights. Reference herein to any specific commercial product, process, or service by trade name, trademark, manufacturer, or otherwise does not necessarily constitute or imply its endorsement, recommendation, or favoring by the United States Government or any agency thereof. The views and opinions of authors expressed herein do not necessarily state or reflect those of the United States Government or any agency thereof. 


\section{DISCLAIMER}

Portions of this document may be illegible in electronic image products. Images are produced from the best available original document. 
We mapped the position of the controlling element Dissociation, Ds, in three instances in which it transposed to $\mathrm{R}$, inhibiting expression of the seed-pigmenting determiner, Sc. Mapping was carried out in heterozygotes between the inhibited alleles and a stable allele conditioning only plant pigmentation ( $P$ determiner). In all three instances $D s$ mapped closely distal to Sc. --- A cyclic pattern of variation which simulates mutation has been established for the allele $\mathrm{R}-\mathrm{g}:$ Peru1304. This allele is stable in vegetative tissues but changes from the strongly expressed original state to a nearly colurless furm in abour 1 per 1000 female gametes. Reversion occurs at about the same frequency in female gametes but three times as frequently in the male germ line. Although restricted to around the stage of meiosis, the change from full color to nearly colorless is not associated with recombination of markers flanking R. - - Previously, we tested for recombinational separability of different tissue-specific determiners with negative results. ( $\underline{S c})$ and $(\underline{P})$, furthermore, recombined similarly with respect to a closely distal marker. In recent tests involving a closely proximal marker, however, recombination occurred with $(\underline{P})$ but not with $(\underline{S c})$. Thus the earlier failure to recover recombination between determiners may not have been due to variation at precisely the same site, but due to the presence of local chromosomal rearrangement which precludes the occurrence of crossing-over or the recovery of recombinants. 
The $\mathrm{R}$ genes in corn exerts precise control over the distribution and the time of appearance of anthocymin pigmentation. L. J. Stadler (1951) described the genetic basis for control in terms of $R$ "determiners" of pigmentation that are tissue and time specific in action. Determiners of a few basic kinds combine in different numbers and arrangements to comprise a wide array of alleles.

For purposes of working out the organization of various alleles in recombination tests, we have been able to treat the "determiners" as point sources of variation. This view probably is overly simplified, certainly so if point source is taken to mean single-site variation. Evidence will be presented in this report that a difference between two determiners is not due to single-site variations even though recombination between the two cannot be demonstrated.

Are there components in addition to the tissue-specific determiners which together comprise a functional $\underline{R}$ unit? Separate lines of evidence sumarized in the following two sections indicate that such is the case. Variation in $\underline{R}$ components other than tissue-determiners may be uncommon in maize races and, when present, overshadowed by the conspicuous nature of determiner differences.

Position of the controlling element (Ds) in relation to the tissue-specific determiners of $R$ action. We previously had isolated three instances in which action of $R-s c$ alleles had come under influence of the transposable controlling element Dissociation, (Ds). The parent R-sc alleles govern strong seed pigmentation and lack plant color effects. Following Ds transposition to R-SC, pigmentation was reduced to colorless or very nearly so in cases $m-3$ and $m-6$, and to faintly pigemented in case m-1. The inhibited phenotypes are stable in the absence of a second element Activator, Ac. In Ac's presence, reversions to full R-sc expression occur, yielding mosaic kernels and frequent germinal reversions to $\underline{R-S C}$. Ds is active only on the $\underline{R}$ allele with which it is coupled in cis linkage phase.

What is the relation of $\underline{D}$ to the component of $\underline{R}$ which determines what plant part will be pigmented? We first tested the inhibited alleles for stability in the absence of Ac. No germinal revertants to R-sc occurred among 41,020 gametes of $\mathrm{m}-\overline{1}$ and 18,179 of $\mathrm{m}-3$. This was the outcome anticipated based on McClintock's (1956) original characterization of $\mathrm{AC}-\mathrm{Ds}$ interaction. Case $\mathrm{m}-6$, however, yielded 16 germinal reversions to $\underline{\mathrm{R}-\mathrm{SC}}$ among 21,620 gametes sampled. This case thus is an exception to the general rule that Ds is "locked" into position in the chromosome in the absence of Ac. Although $m-6$ is of special interest for further investigation, $\overline{\text { it }}$ must be used with due caution in mapping studies since the reversions that were observed in homozygotes are of the same phenotype as products anticipated from recombination between $\underline{D}$ and the determiner of strong seed coloration, (Sc), in appropriate heterozygotes.

Recombination was tested in combinations of the inhibited alleles with a stable one which governs plant pigmentation but is mutant for the seed component $(P)(s)$. The $(P)(s)$ chromosome carried the linked marker genes golden and M-st. Occasional full color kernels, indicating restoration of $(\mathrm{Sc})$ action in $\underline{\mathrm{R}-\mathrm{S}} \mathrm{C}$, were obtained from all three heterozygotes. 
The respective frequencies for $m-1, M-3$ and $m-6$ were $11.0,13.1$ and 9.2 $x 10^{4}$. In the 69 derivatives of $\mathrm{m}-1$ and $\mathrm{m}-3$ for which the linked marker classification is complete, three are marked as the m-1 or m-3 parent whereas the remaining 66 are recombinants. The recombinants are all of one class, carrying the proximal marker of $\mathrm{m}-1$ or $\mathrm{m}-3$ and the distal number of $(P)(s)$. Clearly, in these two cases Ds lies distal to (Sc).

In the case of $m-6$, about one-half of the colored seed derivatives are recombinant, marked as in the case of $\mathrm{m}-1$ and $\mathrm{m}-3$. Most of the remainder are marked as parental $\mathrm{m}-6$. This second class may be the counterpart of $\underline{R-s c}$ reversions obtained from $m-6$ homozygotes. The frequency of recombinant $\mathrm{R}-\mathrm{sc}$ derivation from $\mathrm{m}-6$ may mean that $\mathrm{Ds}$ lies closer to $(\mathrm{Sc})$ in this case than in the case of insertions $\mathrm{m}-1$ and $\mathrm{m}-3$.

The fact that the (P) (s) allele can contribute by recombination a normal function to a Ds inhibited $\underline{R-s c}$, thereby restoring typical $\underline{R-S C}$ action, signals the presence of a componet of $\underline{R}$ in addition to the tissue-specific determiner, i.e. (Sc) or ( $P$ ). This second component evidently is common to $(\mathrm{SC})$ and $(P)$ and lies distal to them. Insertion of $\mathrm{Ds}$ is believed to interrupt continuity between the two cis acting components, arresting gene activity.

If Ds can be situated at different positions between the two components, it should be possible to map the positions of different insertions relative to one another. If a crossover occurred between the two, one product would carry both insertions. The two undoubtedly would inhibit (Sc) expression, yielding a product which cannot be distinguished from the parental forms by present means. The reciprocal product, however, should carry neither $\mathrm{Ds}$ and be readily detected as a functional R-sc derivative. Linked markers arc being iucorporated into the $m-1, \overline{m-3}$, and m-6 chromosomes with a view to conducting this experiment. As detailed further in the current research proposal, we hope to obtain additional instances of $\underline{D s}$ insertion at $\underline{R}$ in order to delimit the entire cisinteracting $(\overline{\mathrm{SC}})$ region.

A cyclic pattern of variation involving $R-g$ :Peru1304. This allele is exceptional among those of the R-sc class in that it yields nearly colorless variants $(r-g: N c)$ in a frequency of nearly one per thousand gametes (Bray and Brink, 1966). We previously tested the notion that such variants were not the result of mutation in the strict sense but rather recombination involving different members of a serial, tandem duplication. If organized as a serial duplication, giving the possibility of $\mathrm{r}-\mathrm{g}$ :Nc origination through unequal crossingover, such variants from R-g:Perul304 homozygotes should be associated with the recombination of linked markers. Such proved not to be the case.

Our more recent investigations were prompted by the observation that $\mathrm{r}-\mathrm{g}: \mathrm{Nc}$ isolates were reverting in turn to a form similar if not identical to parental $\mathrm{R}-\mathrm{g}$ :Peru1304. This behavior had not been established previously for colorless or near-colorless variants except those under Influence of transposable controlling elements. Our determination of $\mathrm{r}-\mathrm{g}: \mathrm{Nc}$ to $\mathrm{R}-\mathrm{g}$ reversion in frequency of $12.1 \times 10^{-4}$ is within the limits of values reported by Bray and Brink for the "forward" direction in the cycle. 
We sought means of testing whether this variation was due to crossingover but of such a nature as not to lead to recombination of flanking markers. One reason for reexamining the role of recombination was the restriction of switching between the two states to about the time of meiosis - there being no evidence of instability in vegetative tissues. Ideally, one would compare reversion frequency in the normal circumstance with a situation where crossing-over is blocked. Unfortunately, there is at present no known means for blocking crossing-over in maize. Recombination rates often differ, however, in the two sexes. Thus through reciprocal crosses we obtained an estimte of the frequency in the male which could be compared with $12.1 \times 10^{-4}$, the value cited above for tests through the female.

$10^{-4}$ Our estimate of $\mathrm{r}-\mathrm{g}: \mathrm{Nc}$ to $\mathrm{R}-\mathrm{g}$ reversion in the male comes to $36.4 \mathrm{x}$ $10^{-4}$ (based on 123 cases,) a three-fold increase relative to the female. One possible basis for the elevated frequency through the male would be the occurrence or premeiotic reversions, yielding clones of germinal tissue. Such events would yield clusters of variants. Yet, when our data are analyzed in terms of the number of variant kernels per ear compared to a random distribution described by Poisson theory, there is not a significant departure from randomness.

A weakness of our present data is the lack of closely comparable $\delta$ vs. $\$$ data for the immediate $\underline{R}$ region. Our several previous analyses involving intralous recombination have all involved the use of the female parent as the test heterozygote. We now have stocks appropriate for testing recombination between $(P)$ and $(S)$ components of the R-r:standard allele through the male. The test involves the use of a combination of recessive mutants in addition to linked markers such that any ralse cases resulting from foreign, contaminant pollen can be positively excluded.

One model for an off and on cyclic of gene expression associated with crossing-over depends on rearrangement within a region of reversed duplication (reversed repeat). We began developing this possibility in more detail after obtaining evidence on the bifunctional nature of given

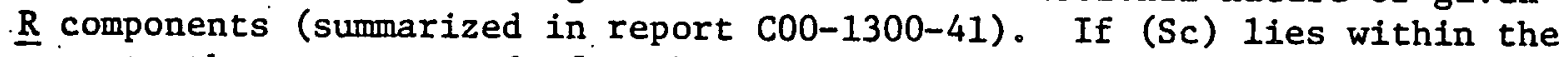
repeat, the sense strands for the two copies have opposite polarities. Recombination within the reverse repeat will not in this case alter the relationship with some second element of the cis-specific region. If one copy of (Sc) were mutant, however, switshing between the normal and mutant copies would result in on and of cycles.

The results reported in the preceding section of this report encourage us to pursue experiments pertaining to the reverse-repeat model for two reasons. First, there is the recombination evidence for cis interacting components of $\mathrm{R}$ actions. Second, this model provides a possible basis for the instability of $\mathrm{m}-6$, supposing that Ds in this instance may be located within the region of reversed duplication. If this were so then one would predict that the noncrossover $\underline{R-s C}$ revertants from it should switch cyclically to the m-6 state. 
Nature of the difference.between the tissue-specific determiners $(\mathrm{Sc})$ and $(\mathrm{P})$.. One of the most unyielding issues in the genetic analysis of $R$ organization has been the basis for tissue-specific control of pigmentation. A given allele governs pigmentation of a particular combination of plant parts at characteristic times. Pigmentation is dominant to its absence. In heterozygotes, the sum of the separate effects of the two alleles is observed, without specific interactions. In the case of heterozygotes involving the plant pigmenting determiner (P) with (S) (a somewhat weaker seed-pigmenting determiner than (Sc)) a segregational test for allelism was made by screening for gametes which carried both (P) and (S) functions in one chromosome. None was found among 38,400 gametes tested (Dooner and Kermicle, 1974). The absence of recombination between determiners suggests that determiner differences may be viewed as local, point sources of variation. This circumstance has proved useful in working out, for example, the number of $\mathrm{R}$ units in the case of chromosomes that are duplicate for the $\underline{R}$ region. At the same time it seemingly precludes an analysis of the determiners themselves by recombinational fractionation.

An alternative use of crossing-over is to compare the level of recombination between some closely linked marker and different determiners. The R-r;standard chromosome, comprised of a duplication carrying (P) in one member and $(S)$ in the other serves as the archetype in this analysis. We previously mapped the site of difference between $(P)$ and (S) relative to the proximal and distal termini of the duplication junction (Dooner and Kermicle, 1971). The relative genetic distances may be summarized relative to the duplicated segment (solid lines), as

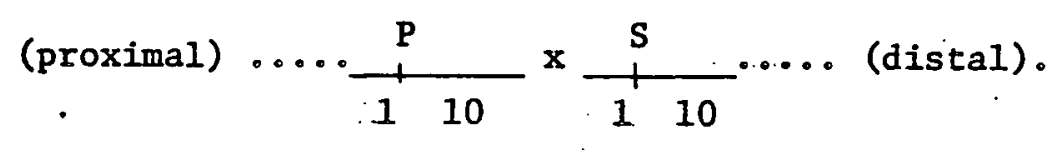

The $(x)$ denotes the junction of the duplication.

In order to test $(\mathrm{Sc})$ relative to the duplication's proximai terminus we incorporated it into the proximal member of a duplication that carried (s) In the distal member. If (Sc) ad (s) occupied the same sites as $(P)$ and $(S)$ then ( $S$ ) derivatives should arise from (Sc) (s) homozygotes by unequal recombiantion. This possibility may be visualized as,

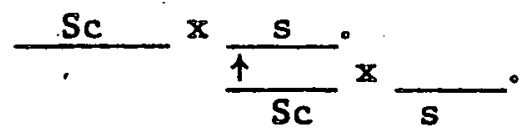

Two putative (s) derivatives were obtained in 106,300 gametes tested. On further analysis both proved however to have retained the duplication. The absences of ( $s$ ) derivatives comparable to (S) cases obtained from (P) (S) homozygotes in earlier tests suggests that ( $\mathrm{Sc}$ ) bears a different relationship to $(S)$ than $(P)$ does to $(S)$.

Next, the $(\mathrm{Sc})(\mathrm{s})$ chromosome was tested in combination with $(\mathrm{P})(\mathrm{s})$ in order to compare more directly the relationships of $(\mathrm{Sc})$ and $(\mathrm{P})$ with (s). If the relationships are the same, chromosomes having the 
properties hoth of (Sc) and of (P) should arise in two, symmetrical fashions, viz.

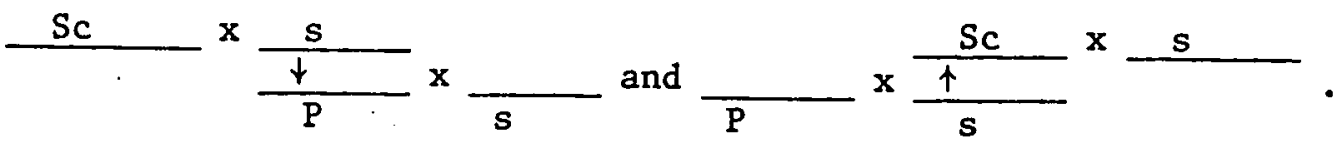

Ten cases having the combined functions of $(\mathrm{Sc})$ and $(\mathrm{P})$ were isolated. All carried the proximal marker of the $(\mathrm{Sc})(\mathrm{s})$ chromosome; eight of the nine cases that were successfully tested for the proximal marker had that of the $(P)(s)$ parent. All eight recombinant chromosomes, that is, were of the linked marker class shown on the left in the above illustration. What accounts for absence of the possibility illustrated to the right?

Conceivably, (Sc) may lie proximally relative to (s) such that displaced pairing of the $(\mathrm{Sc})(\mathrm{s})$ chromosome to the right, i.e.

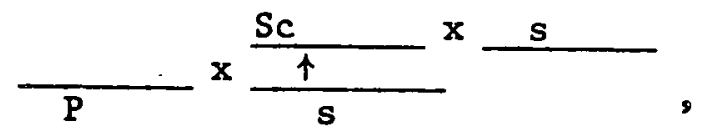

yields $\frac{P}{x} \times \frac{s}{} \quad$ a triplication product that is indistinguishable in phenotypic effects from parental (P) (s). Were this so, however, products of null phenotype should be produced in (P) (S)/(Sc) heterozygotes via $\frac{P}{t} s$ S None were found in a population of $\frac{\downarrow}{\text { Sc }}$

54,900 gametes. That there were 13 crossover (P) deviations (presumed origin via $\frac{P}{t} \times \stackrel{S}{ } \times$ compared to 24 cases in a population of Sc

85,300 gametes of $(P)(S) /(S)$ indicates that recombination between $(P)$ and the duplication junction does not differ appreciably in the two cases.

If the failure to recover (P) (Sc) (s) derivatives from (Sc) (s)/ $(P)(s)$ is due to segmental differentiation of (Sc) relative to ( $s$ ), (P) and $(S)$, then the reciprocal product marked as (s) also should not be recovered. Similarly, the reciprocal of $(S c)(P)(s)$ should be found. We screened accordingly for cases lacking both the seed and plant color effects and established 15 cases in a final seedling population of 79,200. Two exceptions carried the outside markers of parental (P) (s) and four that of $(\mathrm{Sc})(\mathrm{s})$. Of more interest is the nine cases bore on recombinant chromosomes. All but one of these were marked in a manner reciprocal to the eight recombinants obtained from screening for products possessing both $(\mathrm{Sc})$ and $(\mathrm{P})$ functions. In all, therefore, 16 recombinants of one type were recovered compared to one of the second.

The evidence that $(\mathrm{Sc})$ recombines differently than $(\mathrm{P})$ relative to a closely proximal site may be correlated with the results presented in the previous two sections indicating that the ( $\mathrm{Sc}$ ) specificity may be associated with a reversed duplication segment. Is local chromosomal rearrangement 
incidancal or related causually to the tissue-specific action, is a question of obvious interest. We" should now be able to test other determiners in like fashion to see whether local chromosome differentiation is a regular feature of tissue-specific $\underline{R}$ regulation.

\section{LITERATURE CITED}

Bray, R. A. and R. A. Brink. 1966. Mutation and paramutation at the $\underline{R}$ locus in maize. Genetics 54:137-149.

Dooner, H. K. and J. L. Kermicle. 1971. Structure of the $\underline{\mathrm{R}}^{\mathrm{r}}$ tandem duplicatiún In ma1ze. Genetics 67:427-436.

- 1974. Reconstitution of the $\underline{\mathrm{R}}^{\mathrm{r}}$ compound allèle in maize. Genetics 78:691-704.

McClintock, Barbara. 1956. Controlling elements and the gene. Cold Spring Harbor Symp. Quant. Biol. 21:197-216.

Stadler, L. J. 1951. Spontaneous mutation in maize. Cold Spring Harbor Symp. Quant. Bio1. 16:49-64.

\section{CONTRACT PERFORMANCE LEVEL}

Work under this contract has involved participation of the principal investigator at approximately the level indicated in the proposal of July 1976. It is anticipated that participation at this level will continue through the remainder of the contract period even though the University of Wisconsin will be providing an increased proportion of the P.I.'s salary after July 1,1977 . 